International Journal of Engineering \& Technology, 7 (4.13) (2018) 151-154
International Journal of Engineering \& Technology
SPC
Website: www.sciencepubco.com/index.php/IJET
Research paper

\title{
Preliminary investigation on experimental modal analysis of high aspect ratio rectangular wing model
}

\author{
N. A. Rosly, M. Y. Harmin*, D. L. A. A. Majid \\ Department of Aerospace Engineering, Faculty of Engineering, Universiti Putra Malaysia, Malaysia \\ *Corresponding author E-mail: myazdi@upm.edu.my
}

\begin{abstract}
Procedure of conducting an experimental modal analysis (EMA) of roving hammer test for high aspect ratio (HAR) wing containing geometric nonlinearities is presented along with consideration of various tip store sizes. Two sets of test setups of vertical and horizontal arrangements have been considered, which respectively demonstrates the undeformed and deformed cases. Modal properties in terms of natural frequency and mode shape were experimentally measured using the LMS Test.Lab package and the results were then compared between the undeformed and its corresponding deformed configuration. From the finding, it confirms that the chordwise and torsional modes of the undeformed configurations has respectively turned into chordwise-torsion and torsion-chordwise modes as they are in deformed configuration. Meanwhile, the impact related to bending modes is insignificant. Hence, this may result in inaccurate prediction if conventional aeroelastic solution is employed for HAR wing configuration.
\end{abstract}

Keywords: aeroelasticity; experimental modal analysis; geometric nonlinear; high aspect ratio wing; structural dynamics.

\section{Introduction}

Nowadays, current aircraft design has become more flexible and lighter, thus improving its operational range and operating cost per mile. However, an unfortunate consequence to this development is the substantial impact of nonlinear effects, which results in dramatic changes in terms of static and dynamic characteristics of the aircraft. The most cited incidence in this field of study is the crash of NASA pathfinder of Helios prototype, which occurs due to lack of appropriate analyses with respect to the deformed shape of the wing [1]. One of the characteristics of the highly flexible aircraft is geometric nonlinearity, which occurs due to changes in stiffness property as the wing is highly deflected. Moreover, the other main characteristics are due to the force follower effect where the aerodynamic load has to be maintained perpendicular to wing surface deformation. Conventionally, these characteristics are neglected, resulting in inaccuracy in the prediction of both static and dynamic behaviour for highly flexible wing system. Hence, certain degrees of nonlinearity have to be taken into account in order to correctly model the system [2].

The High Altitude Long Endurance (HALE) aircraft can be highly curved even at relatively low aerodynamic loading, hence significant changes in modal characteristics can occur as compared to the straight wing configuration [3]. This will give an impact in terms of bending-torsional coupling, hence causing possibility for aeroelastic behaviour to act differently. A great deal of research works has been devoted to study the effect of nonlinearities with respect to aeroelastic behaviour and most of these studies are focused on developing numerical formulation [4-11]. This includes development of nonlinear reduced order model of combined modal finite element approach and nonlinear beam formulization through displacement based, strain based and intrinsic beam equations.
In the meantime, several research works have been conducted to validate the constructed nonlinear numerical model of high aspect ratio (HAR) wing configuration with the wind tunnel testing. For instance, Tang and Dowell have made a great effort in performing wind tunnel experimental studies on the effects of structural geometrical nonlinearity with respect to flutter boundary, limit cycle oscillation (LCO) and gust responses [12-15]. Their findings show that the developed nonlinear numerical solution is in good agreement with the experimental outcomes. However, the construction of the nonlinear numerical model is rather complex and it is not straightforward to be employed. In their studies as well, a wing store of a slender body is introduced at the wing tip to manipulate the torsional mode for further reduction of the flutter speed to the target envelope of the wind tunnel flutter testing. However, there is still no known experimental modal analysis (EMA) testing that compares the deformed and underformed HAR wing configuration until now. This highlights the research gap that is being addressed by this work.

In this work, the undeformed and deformed configurations represent the wing that is subjected to gravitational loading in spanwise and tranverse bending direction, respectively. The outcome will be asssesed and compared with that of its corresponding undeformed shape in terms of natural frequency and mode shape parameters.

\section{Methodology}

\subsection{Experimental wing model}

An experimental wing model is developed based on the previous parametric study by Rosly and Harmin (2017) [16]. It should be noted that the design space is based upon the wind tunnel specification with consideration of two main criteria upon the selection. The first criterion is the linear flutter speed has to be much lower 
than the maximum operating speed; while the other is the wing tip deflection under gravitational loading has to be lower than half of the cross sectional of the wind tunnel test section. Figure 1 illustrates the basic features of experimental wing model, which consists of a flat plate spar, a set of ribs, fairings and tip store structures, while Table 1 provides the corresponding dimensions. Note that the wing has been modelled to the utmost simple features with relatively simple manufacturing and assembly processes. This will ease any modification and repair whenever required.

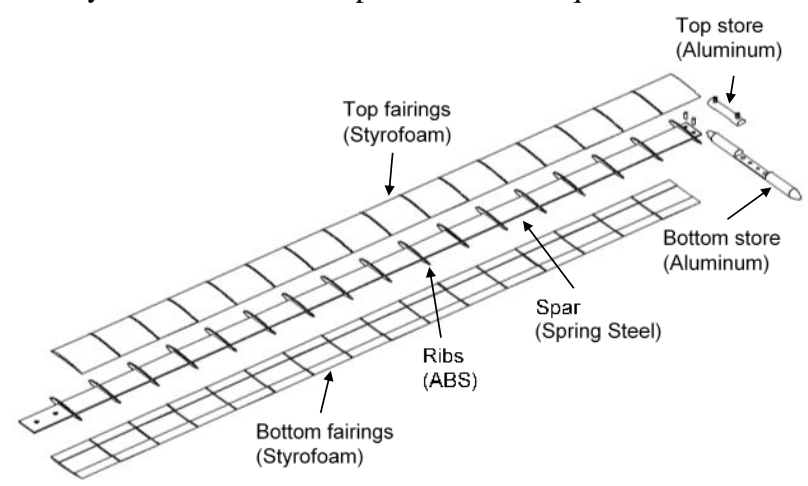

Fig. 1: Exploded wing for experimental wing model

Table 1: Experimental wing model data

\begin{tabular}{|l|c|}
\hline \multicolumn{1}{|c|}{ Properties } & Wing \\
\hline Span & $800 \mathrm{~mm}$ \\
\hline Chord & $50 \mathrm{~mm}$ \\
\hline Spar thickness & $1.25 \mathrm{~mm}$ \\
\hline Centre of gravity & $50 \%$ chord \\
\hline \multicolumn{2}{|c|}{ Store } \\
\hline Radius (m) & $5,7 \mathrm{~mm}$ \\
\hline Length & $150 \mathrm{~mm}$ \\
\hline
\end{tabular}

The spar section of a flat plate is fabricated from spring steel sheet of thickness $1.25 \mathrm{~mm}$ and cut into desired dimension of $800 \mathrm{~mm} \times$ $25 \mathrm{~mm}$. Two holes on both ends of the spar are fabricated, with one end is used for mounting the wing to the clamping mechanism, while the other end is for the attachment of tip store. Both lower and upper parts of the tip store are made from a block of aluminium and they are fabricated using a CNC machine.

The ribs are printed out with 3D printer using Acrylonitrile Butadiene Styrene (ABS) material. They have a shape of NACA 0012 profile, thickness of $1.25 \mathrm{~mm}$ and a slot with cross sectional of spar dimension. Meanwhile, the styrofoam fairings are produced using electrically heated hot wire cutter device, cut from a block of styrofoam. Upon successful manufacturing of all required wing components, they are then assembled in a careful manner. All ribs are slit into the spar and distributed equally along the span with $50 \mathrm{~mm}$ interval between them. The gap between the ribs is covered by the fairings, which complete the aerodynamic contour of the wing. All these wing components are attached permanently with epoxy and Figure 2 provides the depiction of the fully assembled wing model. Note that the ribs arrangement can also be observed in Figure 2.

\subsection{Experimental setup testing}

Experimental modal testing is first carried out on the fully assembled wing, then followed by the wing with store. All structures are tested in vertical and horizontal arrangement. The root of the wing is clamped rigidly to the jig table, which defines a fully-clamped boundary condition. The vertical arrangement provides the undeformed state of the wing structure, which excludes the effect of the gravitational loading on bending direction. On the other hand, the horizontal arrangement provides the deformed state of the wing structure that considered the gravitational effect of $1 \mathrm{~g}$ case.

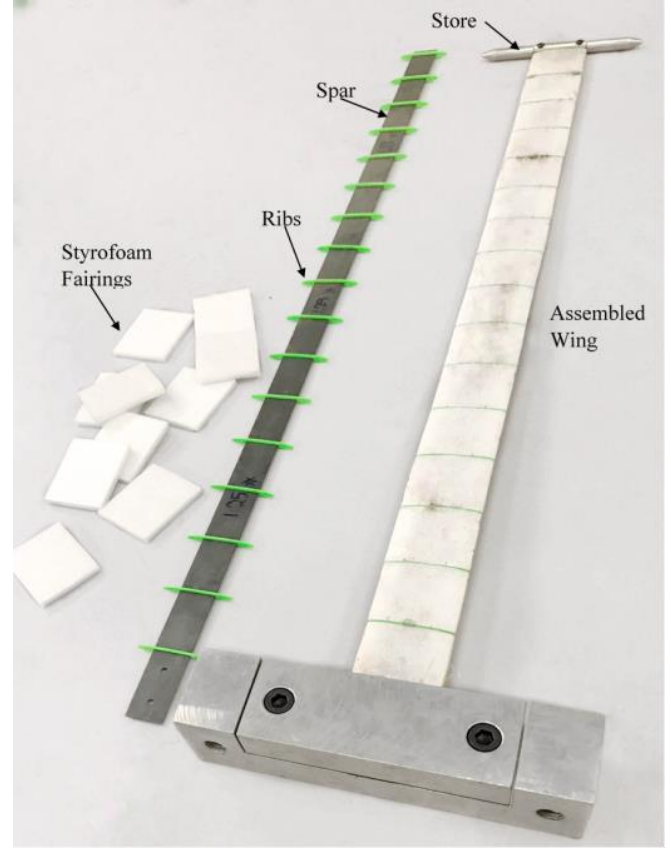

Fig. 2: Components of wing and assembled wing with store

The EMA test setup is consisted of three main components: data analyser (LMS Test.Lab Package), transducer (accelerometer) and excitation mechanism, which can be performed using either shaker or impact hammer excitations. In this work, impact hammer excitation of a roving impact technique is employed due to its attractiveness in ensuring the weight distribution is constant throughout the experimental framework.

Prior to the test, impact points are defined on the ribs at the quarter and three quarter chord along the wing span. This is to enable the mode shape of interest to be captured within the frequency band ranging up to $160 \mathrm{~Hz}$. Two uni-axial accelerometers are attached at two fixed points on the wing in order to measure the dynamic response of the wing structure with their sensitivity of $10.17 \mathrm{mV} / \mathrm{g}$ and $10.23 \mathrm{mV} / \mathrm{g}$, respectively. Figure 3 shows the general setup of considered EMA testing showing, as well as the impact points and direction. These points are impacted three times in flapwise direction for vertical arrangement and in both flapwise and edgewise direction for horizontal arrangement. Following this, the data acquisition and processing system will measure vibration responses of the structure, which are transformed into frequency response functions (FRFs) via Fast Fourier Transformation (FFT) method. The series of FRFs are then analysed in order to extract the natural frequency, damping and corresponding mode shape of the studied structure.

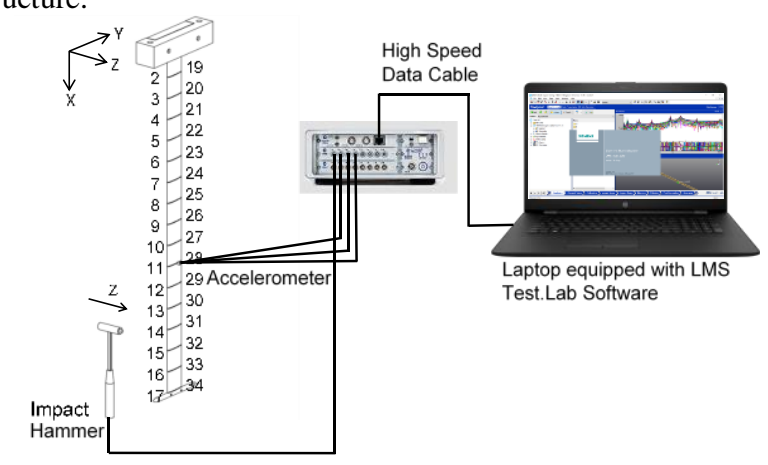

Fig. 3: General setup of experimental modal analysis

\section{Results and discussion}

From a previously conducted study, two torsional related modes of chordwise-torsion and torsion-chordwise modes are expected to be identified for the deformed case [16]. The 'chordwise-torsion' 
implies that the mode is more prominent in the chordwise direction rather than torsional while the 'torsion-chordwise' mode indicates that the mode is more prominent in torsional direction than chordwise. Figure 4 illustrates the sample of measured FRFs for both undeformed and deformed cases for a test setup of uni-axial accelerometer. Note that each of the peaks represents the natural frequency of the system. Nonetheless, the chordwise-torsion mode is unable to be captured due to the dominancy in chordwise shape than its torsional. Hence, this suggests that the test setup has limitation in capturing the mode that is prominent in chordwise direction.

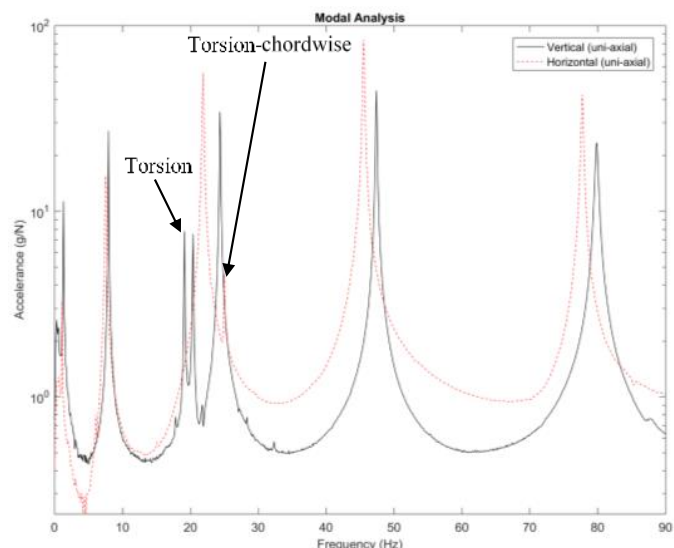

Fig. 4: Measured FRFs for wing with store $14 \mathrm{~mm}$ for vertical and horizontal arrangement using uni-axial accelerometers

Due to this, a tri-axial accelerometer is employed in order to identify both chordwise-torsion and torsion-chordwise modes for the deformed case. Although using tri-axial accelerometer seems like a preferred choice due to its ability to capture all mode shapes of interest, a study by Miaoxian et al. [17] has concluded that adding more references to obtain responses in all degrees of freedom can cause redundancy of data that are prone to errors. Figure 5 illustrates a comparison of deformed case when using uni-axial and triaxial accelerometer test setup. It can be observed that both chordwise-torsion and torsion-chordwise modes are able to be captured via the test setup of the tri-axial accelerometer. Note that there is a slight shifting of frequency peak at a higher frequency as both test setups have different weight distribution due to the different location and weight of the accelerometer. Figure 6 illustrates the mode shapes of wing for both undeformed and deformed wing, showing the bending and torsional related mode shapes over the range of frequency of interest.

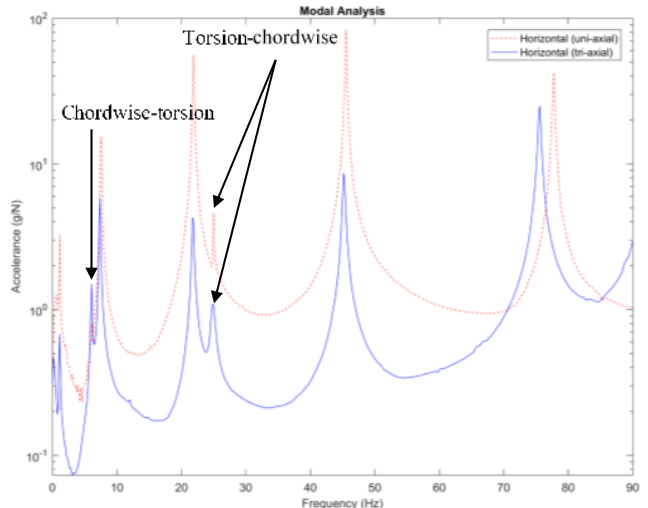

Fig. 5: Measured FRFs for wing with store $14 \mathrm{~mm}$ for horizontal arrangement using uni-axial and tri-axial accelerometers

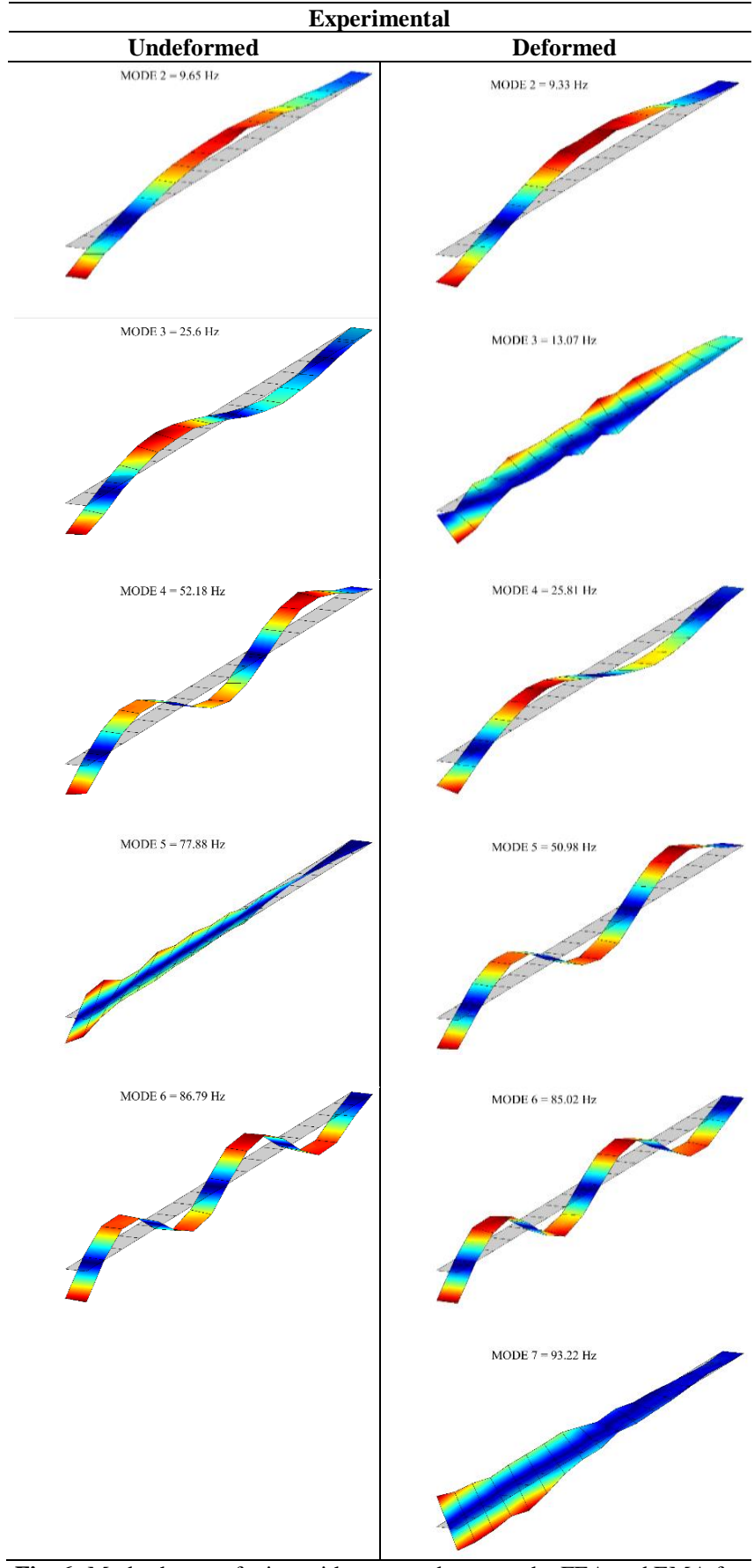

Fig. 6: Mode shapes of wing without store between the FEA and EMA for undeformed configuration.

Table 2 shows a comparison between the EMA of the undeformed and deformed configurations for a wing and wing with stores. For an undeformed configuration, the installation of store gives a significant impact in terms of the torsional mode, as its frequency is reduced with increasing store diameter. On contrary, the impact on bending modes are less affected. This suggests that the installation of store reduces the torsional rigidity of the overall wing system whilst on the other hand maintaining its bending rigidity. It should be noted that the experimental testing is unable to capture the first bending modes due to sensitivity limitation of the accelerometer. However, it can be assumed that the first bending mode will have the same trend as the other bending modes.

In terms of deformed configuration, the trend for bending modes frequencies are more or less similar with the undeformed configuration; whereby the bending frequencies slightly reduces with the increment in store diameter. Note that, within a similar frequency range as in the undeformed case, two torsional related modes are found where they respectively represent the chordwise-torsion and 
torsion-chordwise modes. Overall, it should also be noticed that the torsional-related mode for the deformed case appears to be at a much lower frequency compared to the undeformed case. This is due to changes of overall structure stiffness in the deformed configuration, which in turn replaces the in-plane mode in the undeformed configuration into chordwise-torsion in deformed configuration. This further suggests that the deformed wing in horizontal arrangement behaves differently from undeformed wing in vertical arrangement. Hypothetically, this finding implies that the flutter speed can be further reduced as the torsional-related mode is getting closer to the flutter mode of the second bending modes.

Table 2: Natural frequencies for undeformed and deformed configurations Note: asterisk * refers to torsional mode, the rest refer to bending modes.

\begin{tabular}{|c|c|c|}
\hline Mode & Undeformed & Deformed \\
\hline \multicolumn{3}{|c|}{ Wing } \\
\hline Mode-1 & Unable To Capture & Unable To Capture \\
\hline Mode-2 & 9.65 & 9.33 \\
\hline Mode-3 & 25.60 & $* 13.07$ \\
\hline Mode-4 & 52.18 & 25.81 \\
\hline Mode-5 & $* 77.88$ & 50.98 \\
\hline Mode-6 & 86.79 & 85.02 \\
\hline Mode-7 & & $* * 93.22$ \\
\hline \multicolumn{3}{|c|}{ Wing With Store $10 \mathrm{~mm}$} \\
\hline Mode-1 & Unable To Capture & Unable To Capture \\
\hline Mode-2 & 8.33 & 7.98 \\
\hline Mode-3 & 22.03 & $* 8.43$ \\
\hline Mode-4 & $* 27.77$ & 22.82 \\
\hline Mode-5 & 47.96 & $* * 33.83$ \\
\hline Mode-6 & 80.59 & 46.42 \\
\hline Mode-7 & & 78.24 \\
\hline \multicolumn{3}{|c|}{ Wing With Store $14 \mathrm{~mm}$} \\
\hline Mode-1 & Unable To Capture & Unable To Capture \\
\hline Mode-2 & 7.94 & $* 6.05$ \\
\hline Mode-3 & *19.15 & 7.55 \\
\hline Mode-4 & 24.39 & 21.87 \\
\hline Mode-5 & 47.31 & $* * 25.00$ \\
\hline Mode-6 & 79.85 & 45.54 \\
\hline Mode-7 & & 77.71 \\
\hline
\end{tabular}

\section{Conclusion}

This study is set out to investigate the deformed state of the HAR wing with various store diameters subjected to gravitational loading through EMA. The finding shows that the deformed configuration of HAR wing behaves differently when compared to its corresponding undeformed configuration. This is due to the presence of the chordwise-torsion and torsion-chordwise modes as the wing is deformed in a curve-like shape bending. The other finding shows that the presence of store reduces the torsional rigidity of the wing while maintaining its bending rigidity, which significantly reduces the natural frequencies of torsional mode. Further research should be undertaken to identify the effects of undeformed and deformed configurations with respect to its aeroelastic performance through the wind tunnel flutter testing.

\section{Acknowledgement}

The authors acknowledge the financial support from Ministry of Education (MOE) Malaysia for this study through the Fundamental Research Grant Scheme (FRGS/1/2015/TK09/UPM/02/2) with Project Code: 03-01-15-1625FR.

\section{References}

[1] Noll TE, Brown JM, Perez-Davis ME, Ishmael SD, Tiffany GC \& Gaier M (2004), Investigation of the Helios prototype aircraft mishap. NASA, Langley

[2] Patil MJ, Hodges DH \& Cesnikz CES (1996), Aeroelastic analysis of composite wings. 37th Structures, Structural Dynamics and Materials Conference

[3] Patil MJ, Hodges DH \& Cesnikz CES (1999), Characterizing the effects of geometrical nonlinearities on aeroelastic behavior of high-aspect-ratio wings. International Forum on Aeroelasticity and Structural Dynamics

[4] Peters DA, Karunamoorthy S \& Cao W (1995), Finite state induced flow models. Part I: Two-dimensional thin airfoil. Journal of Aircraft 32(2), 313-322

[5] Su W \& Cesnik CES (2011), Dynamic response of highly flexible flying wings. AIAA Journal 49(2), 324-339

[6] Hesse H \& Palacios R (2014), Reduced-order aeroelastic models for dynamics of maneuvering flexible aircraft. AIAA Journal 52(8), 1717-1732

[7] Catellani M, Cooper JE \& Lemmens Y (2016), Nonlinear static aeroelastic of high aspect ratio wing Aircraft by FEM and multibody methods. 15th Dynamics Specialists Conference.

[8] Harmin MY, Wright JR \& Cooper JE (2011), Aeroelastic behaviour of a wing including geometric nonlinearities. The Aeronautical Journal 115(1174), 767-777

[9] Przekop A \& Rizzi SA (2006), Nonlinear reduced order random response analysis of structures with shallow curvature. AIAA Journal 44, 1767-1778

[10] Tiso P, Jansen E, Abdalla M (2011), Reduction method for finite element nonlinear dynamic analysis of shells. AIAA Journal 49 , 2295-2304

[11] Tang DM \& Dowell EH (2001), Experimental and theoretical study on aeroelastic response of high-aspect-ratio wings. AIAA Journal 39(8), 1430-1441

[12] Tang DM \& Dowell EH (2002), Experimental and theoretical study of gust response for high-aspect-ratio wing. AIAA Journal 40(3), 419-429

[13] Tang DM \& Dowell EH (2002), Limit-cycle hysteresis response for a high-aspect-ratio wing model. Journal of Aircraft 39(5), 885-888

[14] Tang DM \& Dowell EH (2010), Aeroelastic airfoil with free play at angle of attack with gust excitation. AIAA Journal 48(2), 427-442

[15] Gern FH, \& Librescu L (1998), Effects of externally mounted stores on aeroelasticity of advanced swept cantilevered aircraft wings. Aerospace Science and Technology 5, 321-333

[16] Rosly NA \& Harmin MY (2017), Finite element analysis of high aspect ratio wind tunnel wing model: A parametric study. IOP Conf Ser.: Mater. Sci. Eng. 270, 012023

[17] Miaoxian G, Beizhi L, Jianguo Y \& Steven L (2015), Study of experimental modal analysis method of machine tool spindle system. Journal of Vibroengineering 17(6), 3173-3131 